\title{
Analysis of Asynchronous Long-Code Multicarrier CDMA Systems With Correlated Fading
}

\author{
Feng-Tsun Chien, Student Member, IEEE, Chien-Hwa Hwang, Member, IEEE, and C.-C. Jay Kuo, Fellow, IEEE
}

\begin{abstract}
The effects of inter-carrier interference (ICI) and aperiodic random spreading sequences on the performance of asynchronous multicarrier code-division multiple-access (CDMA) systems with correlated fading between sub-carriers are investigated in this research. To conduct theoretical analysis for the maximal ratio combining (MRC) receiver, random parameters including asynchronous delays, correlated Rayleigh fading, and spreading sequences are averaged to find the unconditional covariance matrix of the interference-plus-noise vector. We demonstrate that the ICI in the system proposed by Kondo and Milstein (1996) can be mitigated by assigning a common random spreading sequence over all sub-carriers for each user, rather than using a set of distinct spreading sequences, where code sequences are assumed perfectly time-synchronized. Moreover, the analytic expression for the bit error probability (BEP) can be obtained with the Gaussian approximation. Simulation results are used to demonstrate the accuracy of our analysis. Various design tradeoffs including the number of sub-carriers, fading correlations, ICI and multipath effect are presented in simulation. We conclude that, when properly choosing a sufficiently large number of sub-carriers, the benefit of mitigating multipath interference and exploiting potential hidden frequency diversity shall prevail the impairment brought by increased ICI.
\end{abstract}

Index Terms-Asynchronous transmission, code division multiple access (CDMA), correlated fading, inter-carrier interference (ICI), multicarrier CDMA, random signature sequence.

\section{INTRODUCTION}

$\mathbf{T}$ O COMBINE the advantages of both code-division multiple-access (CDMA) and multicarrier modulation techniques, several multicarrier CDMA systems have been developed over the course of the past decade. The multicarrier-CDMA (MC-CDMA) system was first proposed in [2] to combat the severe indoor multipath effect. Additionally, with a different mechanism of the spreading process, Kondo and Milstein [1] proposed a multicarrier-direct sequence-CDMA

Paper approved by G. E. Corazza, the Editor for Spread Spectrum of the IEEE Communications Society. Manuscript received May 27, 2003; revised February 5,2004 , and October 22, 2004. This work was supported by the Integrated Media Systems Center, a National Science Foundation Engineering Research Center, under Cooperative Agreement EEC-9529152. Any opinions, findings and conclusions or recommendations expressed in this material are those of the authors and do not necessarily reflect those of the National Science Foundation. This paper was presented in part at IEEE Internationl Conference on Communications, Anchorage, AK, May 2003.

F.-T. Chien and C.-C. J. Kuo are with the Integrated Media Systems Center and the Department of Electrical Engineering, Signal and Image Processing Institute, University of Southern California, Los Angeles, CA 90089-2564 USA (e-mail: fchien@usc.edu; cckuo@sipi.usc.edu).

C.-H. Hwang is with the Department of Electrical Engineering and Institute of Communications Engineering, National Tsing Hua University, Hsinchu, Taiwan 30013 R.O.C. (e-mail: chhwang@ee.nthu.edu.tw).

Digital Object Identifier 10.1109/TCOMM.2005.844969
(MC-DS-CDMA) system which is effective in suppressing partial-band interference. Both multicarrier CDMA systems exhibit the capability of mitigating the effect of frequency-selective fading without using the RAKE receiver. Based on these two originating work, a number of multiuser detection schemes applied in the context of multicarrier CDMA systems can also be found in recent publications [3]-[7].

It was assumed in earlier work that each sub-channel in the multicarrier CDMA systems fades independently. In practice, however, this assumption is not generally true. The increase in the number of sub-carriers alleviates channel frequency selectivity on one hand, but reduces the frequency separation between adjacent sub-carriers on the other. The latter may lead to insufficient frequency spacing as compared to channel coherence bandwidth and result in correlated fading, an effect that deteriorates the effectiveness of possible diversity benefit [8]. To analyze the effect of correlated fading between sub-channels, it is required to quantify its statistical characteristics. With a complete description of the multipath intensity profile (MIP) of a wide-sense stationary uncorrelated scattering (WSSUS) channel, the correlation of channel frequency response can be determined by simply taking the Fourier transform of MIP [9].

There have been multiple research studies that examined the effects of correlated fading between sub-channels on the performance of multicarrier communication systems [10]-[14]. Ziemer and Nadgauda [10] averaged the correlated Rayleigh fading coefficients and derived an analytical expression of the average bit error probability (BEP) via finding the eigenvalues of the channel covariance matrix. Based on that, Xu and Milstein [11] evaluated the MC-DS-CDMA system proposed in [1] with more relaxed assumptions, where correlated fading was included and multiple access interference (MAI) as well as partial-band interference were considered. They further proposed multicarrier RAKE systems in [12] to investigate various tradeoffs among the number of sub-carriers, fading correlations and frequency/path diversities. Their extensive simulation results demonstrated that, even with correlated fading, exploiting possible frequency diversity for multicarrier CDMA systems is more advantageous than exploiting possible path diversity of conventional single carrier CDMA systems, when the system experiences a channel with an exponential MIP. Hara and Prasad [13] considered fading correlations in their analysis and the design of an MC-CDMA system. Moreover, Gui and Ng [14] employed the Monte Carlo integration method to obtain the average BEP for generalized asynchronous MC-CDMA systems. While the above mentioned works focused on the analysis of different multicarrier CDMA systems, the impact of 
adopting random signature sequences (long-code) on the BEP performance has not been addressed. The inclusion of aperiodic spreading mechanisms in CDMA systems not only lowers the success probabilities of intentional intercepts but also avoids unpleasant occasions of high correlations between spreading sequences due to asynchronous delays. In view of this, we think it is worthwhile to investigate how the random signature sequences will impact the multicarrier CDMA systems.

In this paper, we extend the work in [10], [11] by considering effects of inter-carrier interference (ICI) and aperiodic random spreading sequences on the performance of asynchronous multicarrier CDMA systems, where the maximal ratio combining (MRC) technique is employed to exploit information among all diversity branches. ICI is one of the major problems in multicarrier systems caused by the loss of orthogonality between sub-carriers. In the situation of severe channel time selectivity or carrier frequency offset, sub-carrier orthogonality may be lost in the process of chip-matched filtering regardless of which shaping waveform, bandlimited or rectangular, is used. Here, we adopt the rectangular chip shaping waveform to analyze the effect of ICI caused by asynchronous transmissions. Based on the Gaussian approximation of MAI [15] and the result in [10], [11], we generalize the expression of average BEP by considering possible repeated roots in finding the generalized eigenvalues of the inverses of the channel and the interference-plus-noise covariance matrices. Finally, tradeoffs between multipath interference and ICI are demonstrated by means of computer simulations.

The rest of this paper is organized as follows. In Section II, the transmitter, channel and receiver models of an asynchronous multicarrier CDMA system are provided. The MRC technique for synchronous and asynchronous systems is discussed in Section III. The corresponding BEP formula is obtained in Section IV. Numerical simulations are presented in Section V. Finally, we provide in Section VI several concluding remarks.

\section{SYSTEM MODEL}

\section{A. Transmitted Signal Model}

Consider the uplink of a multicarrier long-code CDMA system with bandwidth $W$ and $M$ sub-carriers. For both MC-CDMA and MC-DS-CDMA systems, the transmitted signal of user $k$ can be expressed in the complex analytic form as [3]

$$
s_{k}(t)=\sum_{m=1}^{M} \sqrt{\frac{2 P_{k}}{M}} \sum_{i=-\infty}^{\infty} c_{k, m}\left(t-i T_{b}\right) b_{k}(i) e^{j 2 \pi f_{m} t}
$$

where $b_{k}(i)$ denotes the $i$ th data symbol with duration $T_{b}, P_{k}$ the average transmitted power, $c_{k, m}(t)$ the normalized spreading waveform at the $m$ th sub-carrier of user $k$, respectively, and $f_{m}=f_{0}+m(W / M)$ is the modulation frequency at the $m$ th sub-carrier with $f_{0}$ the reference frequency.

The difference between MC-CDMA and MC-DS-CDMA systems lies in the spreading mechanism. In an MC-CDMA system, the data symbol is multiplied by one chip of the signature sequence [2]. In an MC-DS-CDMA system, the data symbol for any given user is modulated by a set of $M$ signature sequences which can be identical or distinct [1], [7], [16]. In MC-DS-CDMA, the spreading waveform $c_{k, m}\left(t-i T_{b}\right)$ in (1) is expressed as

$$
c_{k, m}\left(t-i T_{b}\right)=\sum_{n=0}^{N_{c}-1} c_{k, m}^{(n)}(i) p\left(t-i T_{b}-n T_{c}\right)
$$

where $c_{k, m}^{(n)}(i)$ is the $n$th chip of user $k$ 's signature sequence applied at the $m$ th sub-carrier during the $i$ th symbol interval which takes on values from $\left\{1 / \sqrt{N_{c}},-1 / \sqrt{N_{c}}\right\}$ equally likely, $N_{c}$ is the length of the signature sequence at each sub-carrier, and the time span of the chip-shaping waveform $p(t)$ is equal to one chip interval $T_{c}=T_{b} / N_{c}$. Note that, as implied in (2), we have assumed that signature sequences employed by a particular user over different sub-carriers are time-synchronized, i.e., they have a common switching time.

\section{B. Channel Model}

Let us consider a multicarrier CDMA system with bandwidth $W$ experiencing a WSSUS frequency-selective fading channel with impulse response $h_{k}(\tau ; t)$ for each user $k$, where the signal bandwidth on each sub-carrier is equal to $W / M$. Then, the received signal of a multicarrier CDMA system contributed by the $k$ th user without the effect of noise can be represented as [1], [3]

$$
\begin{aligned}
r_{k}(t)=\sqrt{\frac{2 P_{k}}{M}} \sum_{m=1}^{M} \sum_{i=-\infty}^{\infty} H_{k}\left(f_{m} ; i T_{b}\right) \\
\times c_{k, m}\left(t-i T_{b}\right) b_{k}(i) e^{j 2 \pi f_{m} t}
\end{aligned}
$$

where $H_{k}(f ; t)$ denotes the Fourier transform of $h_{k}(\tau ; t)$. In above, we assume that channel time selectivity is not severe so that $H_{k}\left(f_{m} ; i T_{b}\right)$ is unchanged over a time period of $T_{b}$.

To ensure frequency nonselectivity in multicarrier CDMA systems, it is often required to choose a sufficiently large number of sub-carriers. However, this also results in the reduction of frequency separation $\Delta f=W / M$ between adjacent sub-carriers. When the sub-channel bandwidth is less than the channel coherence bandwidth $(\triangle f)_{c}$, i.e., $\triangle f<(\triangle f)_{c}$, the assumption of uncorrelated fading characteristics between sub-channels has to be relaxed. The statistical relation between two fading coefficients experienced by user $k$ at the $m$ th and $n$th sub-carriers can be described by the frequency correlation function defined by

$$
\Phi_{k}\left(f_{m}, f_{n}\right)=\Phi_{k}\left(f_{m}-f_{n}\right)=E\left[H_{k}\left(f_{m} ; t\right) H_{k}^{*}\left(f_{n} ; t\right)\right]
$$

where the first equality holds because of the wide-sense stationary property and the superscript $*$ denotes the complex conjugate. Then, the statistical property of the Rayleigh fading processes among all $M$ sub-carriers for the $k$ th user can be completely described by the following covariance matrix

$$
\mathbf{R}=E\left[\mathbf{H}_{k}(t) \mathbf{H}_{k}^{H}(t)\right]
$$

where we assume identical channel statistical characteristics for all users and $\mathbf{H}_{k}(t)=\left[H_{k}\left(f_{1} ; t\right), \ldots, H_{k}\left(f_{M} ; t\right)\right]^{T}$. Since the fading process $H_{k}(f ; t)$ for user $k$ is the Fourier transform of $h_{k}(\tau ; t)$, the above frequency correlation function $\Phi(f)$ is the 
Fourier transform of the MIP. In this work, the channel is assumed to have an exponential MIP with normalized unit path energy [9]. Thus, we have

$$
E\left[\left|h_{k}(\tau ; t)\right|^{2}\right]=\frac{1}{T_{d}} e^{-\frac{\tau}{T_{d}}} \mathrm{u}(\tau)
$$

where $T_{d}$ is the root-mean-square (rms) delay spread and $\mathrm{u}(\tau)$ is the unit step function. Then, the frequency correlation function becomes

$$
\Phi_{k}\left(f_{m}, f_{n}\right)=\frac{1}{1+j 2 \pi(m-n)\left(W T_{d} / M\right)} .
$$

\section{Received Signal Model}

The received signal of an asynchronous multicarrier CDMA system with $K$ simultaneous users is given by

$$
r(t)=\sum_{k=1}^{K} r_{k}\left(t-\tau_{k}\right)+n(t)
$$

where $\tau_{k} \in\left[0, T_{b}\right)$ denotes the transmission delay for user $k$, $n(t)$ is the complex additive white Gaussian noise (AWGN) with $E\left[n(t) n^{*}(s)\right]=2 N_{0} \cdot \delta(t-s)$, and $N_{0}$ is the single-sided power spectral density. Without loss of generality, the first user is chosen to be the user of interest. We also assume the receiver has a perfect timing synchronization for this target user, i.e., $\tau_{1}=0$. The propagation delay $\tau_{k}$ for each interfering user can be modeled as $\tau_{k}=\left(u_{k}+v_{k}\right) \cdot T_{c}$, where $u_{k} \in$ $\left\{0,1, \ldots, N_{c}-1\right\}$ and $v_{k} \in[0,1)$ are uniformly distributed random variables. As shown in Fig. 1, the received signal is first fed in parallel to $M$ downconverters, correlated with the chip-shaping waveform $p(t)$, and then sampled at the chip rate. At the $m$ th sub-carrier branch, the received signal vector during the $i$ th observation interval after collecting $N_{c}$ chips can be represented as

$$
\begin{aligned}
\mathbf{r}_{m}(i)= & {\left[r_{m}^{(0)}(i), r_{m}^{(1)}(i), \ldots, r_{m}^{\left(N_{c}-1\right)}(i)\right]^{T} } \\
= & \sum_{k=1}^{K} A_{k} \cdot\left(b_{k}(i-1) \sum_{r=1}^{M} \alpha_{k, r}(i-1) \mathbf{c}_{k, m, r}^{L}(i-1)\right. \\
& \left.+b_{k}(i) \sum_{r=1}^{M} \alpha_{k, r}(i) \mathbf{c}_{k, m, r}^{R}(i)\right)+\mathbf{n}_{m}(i)
\end{aligned}
$$

where

$$
r_{m}^{(n)}(i)=\int_{i T_{b}+n T_{c}}^{i T_{b}+(n+1) T_{c}} r(t) e^{-j 2 \pi f_{m} t} p^{*}\left(t-i T_{b}-n T_{c}\right) d t
$$

is the $n$th chip-matched filter output, $A_{k}=\sqrt{2 P_{k} / M} T_{c}$ accounts for the amplitude scale factor due to the $k$ th user, $\alpha_{k, r}(i)=H_{k}\left(f_{r} ; i T_{b}\right) e^{-j 2 \pi f_{r} \tau_{k}}$, and $\mathbf{n}_{m}(i)$ is a vector of independent complex Gaussian noise samples, each with zero mean and variance $\sigma^{2}=2 N_{0} T_{c}$. After some straightforward manipulation, (7) can be rewritten in a more compact form as

$$
\begin{array}{r}
\mathbf{r}_{m}(i)=\sum_{k=1}^{K} A_{k} \cdot\left(b_{k}(i-1) \mathbf{S}_{k}^{L}(i-1) \mathbf{T}_{k, m} \mathbf{h}_{k}(i-1)\right. \\
\left.+b_{k}(i) \mathbf{S}_{k}^{R}(i) \mathbf{T}_{k, m} \mathbf{h}_{k}(i)\right)+\mathbf{n}_{m}(i)
\end{array}
$$

where

$$
\begin{aligned}
\mathbf{T}_{k, m} & =\operatorname{diag}\left\{\mathbf{t}_{k, m, 1}, \ldots, \mathbf{t}_{k, m, M}\right\}_{2 M \times M} \\
\mathbf{h}_{k}(i) & =\left[\alpha_{k, 1}(i), \ldots, \alpha_{k, M}(i)\right]^{T} \\
\mathbf{S}_{k}^{L}(i-1) & =\left[\mathbf{S}_{k, 1}^{L}(i-1), \ldots, \mathbf{S}_{k, M}^{L}(i-1)\right]_{N_{c} \times 2 M} \\
\mathbf{S}_{k}^{R}(i) & =\left[\mathbf{S}_{k, 1}^{R}(i), \ldots, \mathbf{S}_{k, M}^{R}(i)\right]_{N_{c} \times 2 M}
\end{aligned}
$$

and $\mathbf{S}_{k, r}^{L}(i-1)$ and $\mathbf{S}_{k, r}^{R}(i)$ are shown in the equation at the bottom of the page, and $\mathbf{t}_{k, m, r}=\left[a_{k, m, r}, b_{k, m, r}\right]^{T}$ with elements

$$
\begin{aligned}
& a_{k, m, r}=\frac{1}{T_{c}} \int_{0}^{v_{k} T_{c}} e^{-j 2 \pi\left(f_{m}-f_{r}\right) t} d t
\end{aligned}
$$

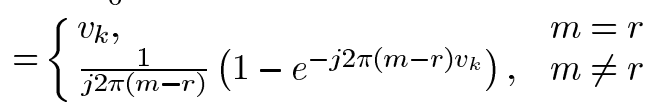

$$
\begin{aligned}
& b_{k, m, r}=\frac{1}{T_{c}} \int_{v_{k} T_{c}}^{T_{c}} e^{-j 2 \pi\left(f_{m}-f_{r}\right) t} d t \\
& = \begin{cases}1-v_{k}, & m=r \\
\frac{-1}{j 2 \pi(m-r)}\left(1-e^{-j 2 \pi(m-r) v_{k}}\right), & m \neq r .\end{cases}
\end{aligned}
$$

Notice that the received signal observed at the $m$ th sub-carrier in (7) contains contributions from not only the $m$ th sub-carrier but also others. The interference from other sub-carriers results from the loss of orthogonality between sub-carriers due to asynchronous transmission. It is commonly referred to as ICI.

\section{MaXimal Ratio Combining Receiver}

\section{A. Synchronous Long-Code Multicarrier CDMA}

We first look at the synchronous transmission case where $\tau_{k}=0$ for all $k$. In this case, the only nonzero $\mathbf{t}_{k, m, r}$ is $\mathbf{t}_{k, m, m}=\left[\begin{array}{ll}0 & 1\end{array}\right]^{T}$ when $r=m$. The received vector $\mathbf{r}_{m}(i)$ in (8) becomes

$$
\mathbf{r}_{m}(i)=\sum_{k=1}^{K} A_{k} \cdot \alpha_{k, m}(i) b_{k}(i) \mathbf{c}_{k, m}(i)+\mathbf{n}_{m}(i)
$$

$$
\begin{aligned}
\mathbf{S}_{k, r}^{L}(i-1) & =\left[\begin{array}{ccccccc}
c_{k, r}^{\left(N_{c}-u_{k}-1\right)}(i-1) & , \ldots, & c_{k, r}^{\left(N_{c}-2\right)}(i-1) & c_{k, r}^{\left(N_{c}-1\right)}(i-1) & 0 & , \ldots, & 0 \\
c_{k, r}^{\left(N_{c}-u_{k}\right)}(i-1) & , \ldots, & c_{k, r}^{\left(N_{c}-1\right)}(i-1) & 0 & 0 & , \ldots, & 0
\end{array}\right]_{2 \times N_{c}}^{T} \\
\mathbf{S}_{k, r}^{R}(i) & =\left[\begin{array}{lllllll}
0 & \ldots, & 0 & 0 & c_{k, r}^{(0)}(i) & , \ldots, & c_{k, r}^{\left(N_{c}-u_{k}-2\right)}(i) \\
0 & , \ldots, & 0 & c_{k, r}^{(0)}(i) & c_{k, r}^{(1)}(i) & , \ldots, & c_{k, r}^{\left(N_{c}-u_{k}-1\right)}(i)
\end{array}\right]_{2 \times N_{c}}^{T}
\end{aligned}
$$




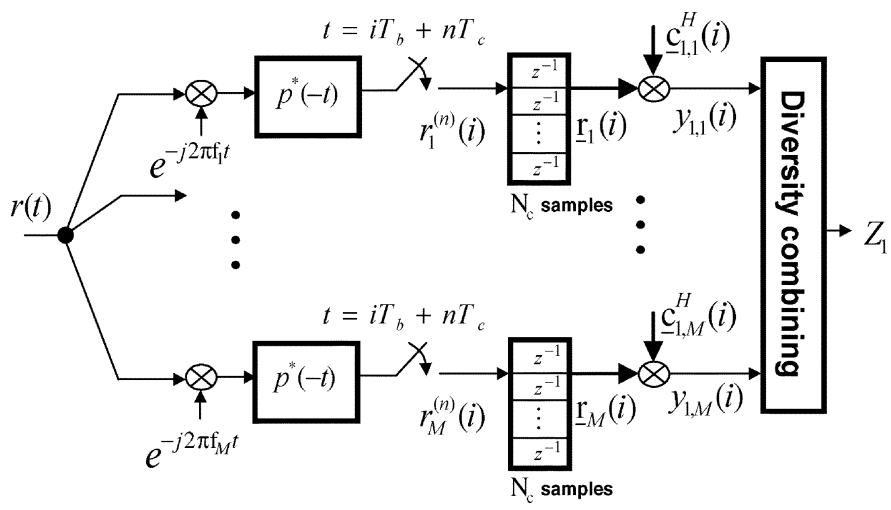

Fig. 1. Chip-matched filter and diversity combining receiver model for the first user.

where $\mathbf{c}_{k, m}(i)=\left[c_{k, m}^{(0)}(i), \ldots, c_{k, m}^{\left(N_{c}-1\right)}(i)\right]^{T}$. Let $y_{1, m}$ be the output after the despreading process at the $m$ th sub-carrier. We have

$$
\begin{aligned}
y_{1, m}(i)= & \mathbf{c}_{1, m}^{H}(i) \mathbf{r}_{m}(i) \\
= & A_{1} \alpha_{1, m}(i) b_{1}(i)+\sum_{k=2}^{K} A_{k} \alpha_{k, m}(i) \rho_{1, k}^{(m)}(i) b_{k}(i) \\
& +\xi_{1, m}(i)
\end{aligned}
$$

where $\rho_{1, k}^{(m)}(i)=\mathbf{c}_{1, m}^{H}(i) \mathbf{c}_{k, m}(i)$ is a random variable representing the cross-correlation between random signature sequences of users 1 and $k$ at the $m$ th sub-carrier with a probability mass function given by

$$
\begin{aligned}
\operatorname{Pr}\left[\rho_{1, k}^{(m)}(i)=\rho\right]= & \left(\begin{array}{c}
N_{c} \\
\frac{N_{c}(\rho+1)}{2}
\end{array}\right) \cdot 2^{-N_{c}}, \\
& \quad \text { for } \quad \rho=-1,-1+\frac{2}{N_{c}}, \ldots,, 1
\end{aligned}
$$

and $\xi_{1, m}(i)=\mathbf{c}_{1, m}^{H}(i) \mathbf{n}_{m}(i)$ is a Gaussian random variable with zero mean and variance $\sigma^{2}$. For the rest of this subsection, we omit symbol index $i$ for the sake of clarity.

Collecting samples from all $M$ sub-carriers, we can write the despread signal in a vector form $\mathbf{y}=\left[y_{1,1}, y_{1,2}, \ldots, y_{1, M}\right]^{T}$ and decompose it into three terms contributed from the desired user, multiple access interference (MAI), and AWGN noise, respectively. That is,

$$
\mathbf{y}=\mathbf{y}_{(1)}+\mathbf{y}_{(\mathrm{MAI})}+\mathbf{n}
$$

where $\mathbf{y}_{(1)}=A_{1} b_{1} \mathbf{h}_{1}, \mathbf{y}_{(\mathrm{MAI})}=\sum_{k=2}^{K} A_{k} b_{k} \mathbf{D}_{k} \mathbf{h}_{k}, \mathbf{n}=$ $\left[\xi_{1,1}, \ldots, \xi_{1, M}\right]^{T}$, and $\mathbf{D}_{k}=\operatorname{diag}\left\{\rho_{1, k}^{(1)}, \ldots, \rho_{1, k}^{(M)}\right\}$.

The MRC technique is capable of exploiting all diversity sources, i.e., despread signals $y_{1, m}$ for $1 \leq m \leq M$, and determines the tapped weighting gains $g_{m}$ 's on all sub-carriers by maximizing the instantaneous signal-to-noise ratio (SNR)

$$
\mathrm{SNR}=\frac{\left|E\left[Z_{1} \mid b_{1}, \mathbf{h}_{1}\right]\right|^{2}}{\operatorname{Var}\left[Z_{1} \mid b_{1}, \mathbf{h}_{1}\right]}
$$

of the output decision statistic $Z_{1}=\sum_{m=1}^{M} g_{m} y_{1, m}$. Finally, the decision is based on the sign of the real part in $Z_{1}$, i.e.,

$$
\hat{b}_{1}=\operatorname{sgn}\left\{\Re\left(Z_{1}\right)\right\} .
$$

Specifically, the weighting vector $\mathrm{g}^{H}=\left[g_{1}, g_{2}, \ldots, g_{M}\right]$ is determined via

$$
\hat{\mathrm{g}}=\arg \max _{\mathbf{g}} \frac{\mathbf{g}^{H} \mathbf{h}_{1} \mathbf{h}_{1}^{H} \mathbf{g}}{\mathbf{g}^{H} \boldsymbol{\Sigma} \mathbf{g}}=\mathbf{\Sigma}^{-1} \mathbf{h}_{1}
$$

where $\boldsymbol{\Sigma}=E\left[\left(\mathbf{y}_{(\mathrm{MAI})}+\mathbf{n}\right)\left(\mathbf{y}_{(\mathrm{MAI})}+\mathbf{n}\right)^{H}\right]$ is the covariance matrix of the interference-plus-noise vector and therefore positive definite. Then, the maximum value of SNR yields

$$
\mathrm{SNR}_{\max }=A_{1}^{2} \cdot \mathbf{h}_{1}^{H} \boldsymbol{\Sigma}^{-1} \mathbf{h}_{1} .
$$

To find the weighting gain in (14), we need an exact expression for $\Sigma$. Given the knowledge of all interfering users' spreading sequences, the conditional covariance matrix of MAI is

$$
E\left[\mathbf{y}_{(\mathrm{MAI})} \mathbf{y}_{(\mathrm{MAI})}^{H} \mid\left\{\mathbf{D}_{k} ; 2 \leq k \leq K\right\}\right]=\sum_{k=2}^{K} A_{k}^{2} \cdot \mathbf{D}_{k} \mathbf{R D}_{k}^{H}
$$

where $\mathbf{R}=E\left[\mathbf{h}_{k} \mathbf{h}_{k}^{H}\right]$ is given in (4). The above expression corresponds to the covariance matrix of a system employing periodic short-codes, where $\mathbf{D}_{k}$ is not a random parameter. In a long-code system where distinct random spreading sequences are assigned to different sub-carriers for each user, as it might be in an MC-CDMA system, we need to average out the random code effect of $\mathbf{D}_{k}$ in (16), which gives

$$
E\left[\mathbf{y}_{(\mathrm{MAI})} \mathbf{y}_{(\mathrm{MAI})}^{H}\right]=\sum_{k=2}^{K} A_{k}^{2} \cdot E\left[\mathbf{D}_{k} \mathbf{R D}_{k}^{H}\right]=\sum_{k=2}^{K} \frac{A_{k}^{2}}{N_{c}} \cdot \mathbf{I}_{M}
$$

where $\mathbf{I}_{M}$ denotes the $M \times M$ identity matrix. On the other hand, in the case where a common random spreading sequence is employed over all sub-carriers for each user [1], we have the unconditional covariance matrix of MAI

$$
E\left[\mathbf{y}_{(\mathrm{MAI})} \mathbf{y}_{(\mathrm{MAI})}^{H}\right]=\sum_{k=2}^{K} \frac{A_{k}^{2}}{N_{c}} \cdot \mathbf{R} \text {. }
$$

\section{B. Asynchronous Long-Code Multicarrier CDMA}

When there exists a random asynchronous transmission delay for each interfering user, the output vector after despreading at the $i$ th symbol interval is given by

$$
\begin{array}{r}
\mathbf{y}(i)=A_{1} b_{1}(i) \mathbf{h}_{1}(i)+\sum_{k=2}^{K} A_{k} \cdot\left(b_{k}(i-1) \mathbf{D}_{k}^{L} \mathbf{T}_{k} \mathbf{h}_{k}(i-1)\right. \\
\left.+b_{k}(i) \mathbf{D}_{k}^{R} \mathbf{T}_{k} \mathbf{h}_{k}(i)\right)+\mathbf{n}(i)
\end{array}
$$

where $\mathbf{T}_{k}=\left[\mathbf{T}_{k, 1}^{T}, \mathbf{T}_{k, 2}^{T}, \ldots, \mathbf{T}_{k, M}^{T}\right]^{T}$, and

$$
\begin{aligned}
& \mathbf{D}_{k}^{L}=\operatorname{diag}\left\{\mathbf{c}_{1,1}^{H}(i) \mathbf{S}_{k}^{L}(i-1), \ldots, \mathbf{c}_{1, M}^{H}(i) \mathbf{S}_{k}^{L}(i-1)\right\}_{M \times 2 M^{2}} \\
& \mathbf{D}_{k}^{R}=\operatorname{diag}\left\{\mathbf{c}_{1,1}^{H}(i) \mathbf{S}_{k}^{R}(i), \ldots, \mathbf{c}_{1, M}^{H}(i) \mathbf{S}_{k}^{R}(i)\right\}_{M \times 2 M^{2}} .
\end{aligned}
$$

Similarly, the first and second terms in (17) correspond to $\mathbf{y}_{(1)}$ and $\mathbf{y}_{(\mathrm{MAI})}$, i.e., contributions from the desired user and MAI, 
respectively. Each element of $\mathbf{y}_{(\mathrm{MAI})}$ also contains interference from other sub-carriers.

The weighting vector $\mathbf{g}$ optimally combining the output statistics from all sub-carriers in the MRC receiver was given previously in (14), which demands the knowledge of $\boldsymbol{\Sigma}$. Given all interferers' spreading codes and asynchronous delays, we have the conditional covariance of $\mathbf{y}_{(\mathrm{MAI})}$ represented by

$$
\begin{aligned}
E & {\left[\mathbf{y}_{(\mathrm{MAI})} \mathbf{y}_{(\mathrm{MAI})}^{H} \mid\left\{\mathbf{D}_{k}^{L}, \mathbf{D}_{k}^{R}, u_{k}, v_{k} ; 2 \leq k \leq K\right\}\right] } \\
& =\sum_{k=2}^{K} A_{k}^{2} \cdot\left(\mathbf{D}_{k}^{L} \mathbf{T}_{k} \mathbf{R}_{h} \mathbf{T}_{k}^{H} \mathbf{D}_{k}^{L^{H}}+\mathbf{D}_{k}^{R} \mathbf{T}_{k} \mathbf{R}_{h} \mathbf{T}_{k}^{H} \mathbf{D}_{k}^{R^{H}}\right)
\end{aligned}
$$

with $\mathbf{R}_{h}=E\left[\mathbf{h}_{k}(i-1) \mathbf{h}_{k}^{H}(i-1)\right]=E\left[\mathbf{h}_{k}(i) \mathbf{h}_{k}^{H}(i)\right]$ in a wide-sense stationary channel. Since $\mathbf{h}_{k}(i)=$ $\left[\alpha_{k, 1}(i), \ldots, \alpha_{k, M}(i)\right]^{T}$ is a composite vector incorporating channel effects and random asynchronous delays of the $k$ th user from all sub-carriers, $\mathbf{R}_{h}$ can be further decomposed as

$$
\mathbf{R}_{h}=\mathbf{V}_{k} \mathbf{R V}_{k}^{H}
$$

where $\mathbf{V}_{k}=\operatorname{diag}\left\{1, e^{-j 2 \pi v_{k}}, \ldots, e^{-j 2 \pi(M-1) v_{k}}\right\}$ and $\mathbf{R}$ is given in (4).

Now, we have separated mutually independent random parameters $\left(u_{k}, v_{k}\right)$ in different matrices. The effect of a shifted random spreading codes resulting from $u_{k}$ 's is considered in $\mathbf{D}_{k}^{L}$ and $\mathbf{D}_{k}^{R}$, while the random asynchronous delays $v_{k}$ 's reside in $\mathbf{T}_{k}$ as well as $\mathbf{V}_{k}$. Our purpose is to average all random terms appearing in (18) and finally derive the unconditional covariance matrix $\boldsymbol{\Sigma}$.

We first deal with the uniformly distributed random variable $v_{k} \in[0,1)$. Since the spreading codes $u_{k}$ and $v_{k}$ are mutually independent, we can take the expectation with respect to $v_{k}$ in (18) and obtain

$$
\begin{gathered}
E\left[\mathbf{y}_{(\mathrm{MAI})} \mathbf{y}_{(\mathrm{MAI})}^{H} \mid\left\{\mathbf{D}_{k}^{L}, \mathbf{D}_{k}^{R}, u_{k} ; 2 \leq k \leq K\right\}\right] \\
=\sum_{k=2}^{K} A_{k}^{2} \cdot\left(\mathbf{D}_{k}^{L} E_{v_{k}}\left[\mathbf{T}_{k} \mathbf{V}_{k} \mathbf{R} \mathbf{V}_{k}^{H} \mathbf{T}_{k}^{H}\right] \mathbf{D}_{k}^{L^{H}}\right. \\
\left.+\mathbf{D}_{k}^{R} E_{v_{k}}\left[\mathbf{T}_{k} \mathbf{V}_{k} \mathbf{R} \mathbf{V}_{k}^{H} \mathbf{T}_{k}^{H}\right] \mathbf{D}_{k}^{R^{H}}\right)
\end{gathered}
$$

which requires finding the expectation of the $2 M^{2} \times 2 M^{2}$ matrix presented at the bottom of the page (written in a block matrix representation).

Let $\mathbf{w}_{k, r}$ be a $2 M \times 1$ vector defined by $\mathbf{w}_{k, r} \triangleq$ $\left[\mathbf{t}_{k, r, 1}^{T}, \mathbf{t}_{k, r, 2}^{T} e^{-j 2 \pi v_{k}}, \ldots, \mathbf{t}_{k, r, M}^{T} e^{-j 2 \pi(M-1) v_{k}}\right]^{T}$ for $1 \leq$ $r \leq M$. Then, the product of $\mathbf{T}_{k, r}$ and $\mathbf{V}_{k, r}$, i.e., $\mathbf{T}_{k, r} \mathbf{V}_{k}$, is a $2 M \times M$ block-diagonal matrix with $\mathbf{t}_{k, r, r^{\prime}}^{T} e^{-j 2 \pi\left(r^{\prime}-1\right) v_{k}}$ being its $r^{\prime}$ th diagonal block for $1 \leq r, r^{\prime} \leq M$. Based on this block-diagonal structure, we obtain the $2 M \times 2 M$ block matrix at the $(m, n)$ th block location in (20) at the bottom of the page as

$$
\begin{aligned}
E_{v_{k}}\left[\mathbf{T}_{k, m} \mathbf{V}_{k} \mathbf{R} \mathbf{V}_{k}^{H} \mathbf{T}_{k, n}^{H}\right] & =E_{v_{k}}\left[\mathbf{R} \odot \mathbf{w}_{k, m} \mathbf{w}_{k, n}^{H}\right] \\
& =\mathbf{R} \odot \mathbf{W}_{m, n}
\end{aligned}
$$

where $\odot$ denotes the block Hadamard product and $\mathbf{W}_{m, n}=$ $E\left[\mathbf{w}_{k, m} \mathbf{w}_{k, n}^{H}\right]$. The definition of the block Hadamard product is given in the Appendix. Specifically, the $(p, q)$ th $2 \times 2$ block matrix of (21) is obtained from the $(p, q)$ th $2 \times 2$ block of $\mathbf{W}_{m, n}$ multiplied with the $(p, q)$ th element of $\mathbf{R}$, i.e.,

$$
\left(\mathbf{R} \odot \mathbf{W}_{m, n}\right)_{p, q}=\mathbf{R}_{p, q} \cdot\left(\mathbf{W}_{m, n}\right)_{p, q} \cdot
$$

Also, note that the $2 \times 2$ block matrix $\left(\mathbf{W}_{m, n}\right)_{p, q}$ located at the $(p, q)$ th block of $\mathbf{W}_{m, n}$ can be explicitly evaluated via

$$
\left(\mathbf{W}_{m, n}\right)_{p, q}=E\left[\mathbf{t}_{k, m, p} \mathbf{t}_{k, n, q}^{H} e^{-j 2 \pi(p-q) v_{k}}\right]
$$

for $1 \leq p, q \leq M$, whose complete analytic results corresponding to all possible combinations of $(m, n)$ and $(p, q)$ are provided in the Appendix.

In a periodic short-code multicarrier CDMA system, we need to find the exact averaged value of all block matrices appearing in (20). However, in a long-code system, only the diagonal elements of each block matrix are of primary interest due to the uncorrelated statistical property between any shifted version of spreading sequences, which will be discussed below.

Let us consider the effect of random spreading sequences contributed by $\mathbf{D}_{k}^{L}$ and $\mathbf{D}_{k}^{R}$. In either case of an MC-DS-CDMA system employing identical or distinct random spreading sequences over different sub-carriers for a given user, $\mathbf{D}_{k}^{L}$ and $\mathbf{D}_{k}^{R}$ are uncorrelated. Thus, by taking the expectation of (19) with respect to $\mathbf{D}_{k}^{L}$ and $\mathbf{D}_{k}^{R}$, we obtain

$$
\begin{aligned}
& E\left[\mathbf{y}_{(\mathrm{MAI})} \mathbf{y}_{(\mathrm{MAI})}^{H} \mid\left\{u_{k} ; 2 \leq k \leq K\right\}\right] \\
& \quad=\sum_{k=2}^{K} A_{k}^{2} \cdot E_{\left\{\mathbf{D}_{k}^{L}, \mathbf{D}_{k}^{R}\right\}}\left[\mathbf{D}_{k} E_{v_{k}}\left[\mathbf{T}_{k} \mathbf{V}_{k} \mathbf{R} \mathbf{V}_{k}^{H} \mathbf{T}_{k}^{H}\right] \mathbf{D}_{k}^{H}\right]
\end{aligned}
$$

where $\mathbf{D}_{k}=\mathbf{D}_{k}^{R}+\mathbf{D}_{k}^{L}$ and we have added two zero terms, $E_{\left\{\mathbf{D}_{k}^{L}, \mathbf{D}_{k}^{R}\right\}}\left[\mathbf{D}_{k}^{L} E_{v_{k}}\left[\mathbf{T}_{k} \mathbf{V}_{k} \mathbf{R} \mathbf{V}_{k}^{H} \mathbf{T}_{k}^{H}\right] \mathbf{D}_{k}^{R^{H}}\right]$ and its Hermitian, without affecting the result. Define a cross-correlation vector

$$
\mathbf{d}_{k, r}^{H} \triangleq \mathbf{c}_{1, r}^{H}\left[\mathbf{S}_{k}^{L}(i-1)+\mathbf{S}_{k}^{R}(i)\right], \quad 1 \leq r \leq M .
$$

$$
E_{v_{k}}\left[\mathbf{T}_{k} \mathbf{V}_{k} \mathbf{R} \mathbf{V}_{k}^{H} \mathbf{T}_{k}^{H}\right]=\left[\begin{array}{cccc}
E_{v_{k}}\left[\mathbf{T}_{k, 1} \mathbf{V}_{k} \mathbf{R} \mathbf{V}_{k}^{H} \mathbf{T}_{k, 1}^{H}\right] & E_{v_{k}}\left[\mathbf{T}_{k, 1} \mathbf{V}_{k} \mathbf{R} \mathbf{V}_{k}^{H} \mathbf{T}_{k, 2}^{H}\right] & , \ldots, & E_{v_{k}}\left[\mathbf{T}_{k, 1} \mathbf{V}_{k} \mathbf{R} \mathbf{V}_{k}^{H} \mathbf{T}_{k, M}^{H}\right] \\
E_{v_{k}}\left[\mathbf{T}_{k, 2} \mathbf{V}_{k} \mathbf{R} \mathbf{V}_{k}^{H} \mathbf{T}_{k, 1}^{H}\right] & E_{v_{k}}\left[\mathbf{T}_{k, 2} \mathbf{V}_{k} \mathbf{R} \mathbf{V}_{k}^{H} \mathbf{T}_{k, 2}^{H}\right] & \ldots, & \vdots \\
\vdots & \vdots & \ddots & \vdots \\
E_{v_{k}}\left[\mathbf{T}_{k, M} \mathbf{V}_{k} \mathbf{R} \mathbf{V}_{k}^{H} \mathbf{T}_{k, 1}^{H}\right] & , \ldots, & , \ldots, & E_{v_{k}}\left[\mathbf{T}_{k, M} \mathbf{V}_{k} \mathbf{R} \mathbf{V}_{k}^{H} \mathbf{T}_{k, M}^{H}\right]
\end{array}\right]
$$


Each element of $\mathbf{d}_{k, r}$ is a discrete random variable with the probability mass function given in (10) and corresponds to a cross-correlation between the spreading codes of the desired user and a shifted version of the interfering user at the $r$ th subcarrier. Then, the $(m, n)$ th element of (23) can be evaluated via

$$
\begin{aligned}
E\left[\mathbf{y}_{(\mathrm{MAI})} \mathbf{y}_{(\mathrm{MAI})}^{H} \mid\left\{u_{k} ; 2 \leq k \leq K\right\}\right]_{m, n} \\
=\sum_{k=2}^{K} A_{k}^{2} \cdot E\left[\mathbf{d}_{k, m}^{H}\left(\mathbf{R} \odot \mathbf{W}_{m, n}\right) \mathbf{d}_{k, n}\right] .
\end{aligned}
$$

For an MC-DS-CDMA system employing a common random spreading code over all sub-carriers for a given user [1], the covariance matrix of the cross-correlation vector is given by

$$
E\left[\mathbf{d}_{k, r} \mathbf{d}_{k, r^{\prime}}^{H}\right]=\frac{1}{N_{c}} \mathbf{1}_{M \times M} \otimes \mathbf{I}_{2}, \quad 1 \leq r, r^{\prime} \leq M
$$

where $\mathbf{1}_{M \times M}$ represents the $M \times M$ matrix with all elements equal to 1 , and $\otimes$ denotes the standard Kronecker product. In this case, the expectation in (24) is found to be

$$
\begin{aligned}
E\left[\mathbf{d}_{k, m}^{H}\left(\mathbf{R} \odot \mathbf{W}_{m, n}\right) \mathbf{d}_{k, n}\right] & \\
& =\frac{1}{N_{c}} \sum_{p=1}^{M} \sum_{q=1}^{M} \mathbf{R}_{p, q} \cdot \operatorname{tr}\left\{\left(\mathbf{W}_{m, n}\right)_{p, q}\right\}
\end{aligned}
$$

where $\operatorname{tr}\{\cdot\}$ denotes the matrix trace operator. Details of the derivation of (26) are provided in the Appendix. The terms with $m \neq n$ and $p \neq q$ correspond to the influence of correlations, including ICI and correlated fading processes, between any two sub-carriers. The results of $\left(\mathbf{W}_{m, n}\right)_{p, q}$ for each possible pairs of $(m, n)$ and $(p, q)$ are also given in the Appendix, and the diagonal $(m=n)$ and off-diagonal $(m \neq n)$ elements of (24) can be derived, respectively, as

$$
\begin{aligned}
E\left[\mathbf{d}_{k, m}^{H}\left(\mathbf{R} \odot \mathbf{W}_{m, m}\right) \mathbf{d}_{k, m}\right] & \\
=\frac{1}{N_{c}} & {\left[\frac{2}{3}+\sum_{\substack{p=1 \\
p \neq m}}^{M} \frac{1}{\pi^{2}(m-p)^{2}}-\sum_{\substack{p=1 \\
p \neq m}}^{M} \frac{1}{\pi^{2}(m-p)^{2}} \Re\left(\mathbf{R}_{p, n}\right)\right.} \\
& \left.+\sum_{\substack{p, q=1 \\
p \neq q \neq m}}^{M} \frac{1}{\pi^{2}(m-p)(m-q)} \Re\left(\mathbf{R}_{p, q}\right)\right]
\end{aligned}
$$

and

$$
\begin{aligned}
E\left[\mathbf{d}_{k, m}^{H}\left(\mathbf{R} \odot \mathbf{W}_{m, n}\right) \mathbf{d}_{k, n}\right] & =\frac{1}{\pi^{2} N_{c}}\left[\frac{-1}{(m-n)^{2}}+\sum_{\substack{p=1 \\
p \neq m \neq n}}^{M} \frac{1}{2(m-p)(n-p)}\right. \\
& +\frac{2}{(m-n)^{2}} \Re\left(\mathbf{R}_{m, n}\right)+\frac{1}{m-n} \\
& \left.\times \sum_{\substack{p=1 \\
p \neq m \neq n}}^{M}\left(\frac{1}{p-n} \Re\left(\mathbf{R}_{p, n}\right)+\frac{1}{m-p} \Re\left(\mathbf{R}_{m, p}\right)\right)\right] .
\end{aligned}
$$

On the other hand, in a system employing different spreading codes at distinct sub-carriers for a given user, cross-correlation vectors from different sub-carriers are uncorrelated, yielding

$$
E\left[\mathbf{d}_{k, r} \mathbf{d}_{k, r^{\prime}}^{H}\right]=\frac{1}{N_{c}} \mathbf{I}_{2 M} \cdot \delta_{r, r^{\prime}}, \quad 1 \leq r, r^{\prime} \leq M
$$

where $\delta_{r, r^{\prime}}$ is the Kronecker delta function. Intuitively, the effect of correlated statistics among sub-carriers is eliminated in this case due to uncorrelated random spreading codes, leaving only the diagonal terms in (24) nonzero. To see this, the result of (26) now becomes

$$
E\left[\mathbf{d}_{k, m}^{H}\left(\mathbf{R} \odot \mathbf{W}_{m, n}\right) \mathbf{d}_{k, n}\right]=\frac{1}{N_{c}} \operatorname{tr}\left\{\mathbf{R} \odot \mathbf{W}_{m, n}\right\} \cdot \delta_{m, n}
$$

where, by using (22), the value of the trace in the above equation is given by

$$
\operatorname{tr}\left\{\mathbf{R} \odot \mathbf{W}_{m, m}\right\}=\frac{2}{3}+\sum_{p=1, p \neq m}^{M} \frac{1}{\pi^{2}(m-p)^{2}}
$$

where $\mathbf{R}_{p, p}=\mathbf{R}_{m, m}=1$ since a normalized unit path energy is considered. At the $m$ th sub-carrier, the first and second components in (28) correspond to the average power contribution of MAI from its own sub-carrier and from other sub-carriers, respectively.

Finally, the last random parameter needed to be averaged out is the discrete random variable $u_{k}$ taking values from $\left\{0,1, \ldots, N_{c}-1\right\}$ with equal probability. For each possible occurrence of $u_{k}$, the probability distribution of the cross-correlation vector $\mathbf{d}_{k, r}$, for all $k \geq 2$ and $r$, is the same. We can conclude immediately that the covariance matrix of $\mathbf{y}_{(\mathrm{MAI})}$ is just equal to that obtained in (24).

\section{ANALYSIS OF BIT ERROR PROBABILITY}

Consider the case of independent binary symbols $\left\{b_{1}(i)\right\}$ with an equal priori probability. Given the decision rule established in (13) and the Gaussian assumption on the statistics contributed from MAI and noise, the approximated BEP of the desired user conditioned on its fading coefficients $\mathbf{h}_{1}$ can be expressed as

$$
P_{e \mid \mathbf{h}_{1}}=Q\left(\sqrt{2 \mathrm{SNR}_{\max }}\right)
$$

where $\mathrm{SNR}_{\max }=A_{1}^{2} \cdot \mathbf{h}_{1}^{H} \boldsymbol{\Sigma}^{-1} \mathbf{h}_{1}$ as given in (15). By defining $\mathbf{x}=\sqrt{2} A_{1} \mathbf{h}_{1}$, we have the unconditional BEP

$$
P_{e}=E_{\mathbf{x}}\left[P_{e \mid \mathbf{x}}\right]=E_{\mathbf{x}}\left[Q\left(\sqrt{\left.\mathbf{x}^{H \Sigma^{-1} \mathbf{x}}\right)}\right]\right.
$$

To start with, we determine the distribution of $\mathbf{x}^{H} \Sigma^{-1} \mathbf{x}$, where the probability density function of $\mathbf{x}$ is

$$
p_{\mathbf{x}}(\mathbf{x})=\frac{1}{\pi^{M} \operatorname{det}\left(\mathbf{R}_{x}\right)} \exp \left(-\mathbf{x}^{H} \mathbf{R}_{x}^{-1} x\right)
$$


with $\mathbf{R}_{x}=E\left[\mathbf{x x}^{H}\right]=2 A_{1}^{2} \mathbf{R}$ denoting the covariance matrix of $\mathbf{x}$. Let $z=\mathbf{x}^{H} \Sigma^{-1} \mathbf{x}$. Then, the moment generating function (mgf) of $z$ is

$$
\begin{aligned}
M_{z}(s) & =E\left[e^{s z}\right] \\
& =\frac{1}{\operatorname{det}\left(\mathbf{I}-s \mathbf{R}_{x} \Sigma^{-1}\right)}=\prod_{i=1}^{N_{e}} \frac{1}{\left(1-s \lambda_{i}\right)^{M_{i}}}
\end{aligned}
$$

where $\left\{\lambda_{i}, i=1, \ldots, N_{e},\right\}$ is the set of distinct eigenvalues, each with multiplicity $M_{i}$, of the matrix $\mathbf{R}_{x} \Sigma^{-1}$ and $\sum_{i=1}^{N_{e}} M_{i}=M$.

If all the eigenvalues of $\mathbf{R}_{x} \Sigma^{-1}$ are identical, e.g., synchronous transmission with uncorrelated fading processes and random spreading sequences, the mgf of (30) becomes

$$
M_{z}(s)=\frac{1}{(1-s \lambda)^{M}}
$$

which corresponds to the mgf of a scaled chi-squared random variable with $2 M$ degrees of freedom. This leads to an averaged BEP of (29) given by [17]

$$
\begin{aligned}
P_{e} & =\frac{1}{2}\left[1-\frac{1}{\sqrt{1+\frac{2}{\lambda}}}\left(1+\sum_{n=1}^{M-1} \frac{1 \cdot 3 \cdot 5 \cdots(2 n-1)}{n ! 2^{n}\left(\frac{\lambda}{2}+1\right)^{n}}\right)\right] \\
& \triangleq P(\lambda, M) .
\end{aligned}
$$

In general, eigenvalues of $\mathbf{R}_{x} \Sigma^{-1}$ are not necessarily all identical or all distinct. By partial fractional expansion, the mgf of (30) can be decomposed to

$$
M_{z}(s)=\prod_{i=1}^{N_{e}} \frac{1}{\left(1-s \lambda_{i}\right)^{M_{i}}}=\sum_{i=1}^{N_{e}} \sum_{m=1}^{M_{i}} \frac{\beta_{i, m}}{\left(1-s \lambda_{i}\right)^{m}}
$$

where

$$
\beta_{i, m}=\frac{\left(-\lambda_{i}^{-1}\right)^{M_{i}-m}}{\left(M_{i}-m\right) !} f_{i}^{\left(M_{i}-m\right)}\left(-\lambda_{i}^{-1}\right)
$$

with $f_{i}(s)=\left(1-s \lambda_{i}\right)^{M_{i}} M_{z}(s)$ and $f_{i}^{(n)}(s)$ its $n$th derivative. Consequently, after taking the inverse Laplace transform, the pdf of $z$ can be represented as a sum of pdf's of scaled chisquared random variables, yielding an averaged BEP

$$
P_{e}=\sum_{i=1}^{N_{e}} \sum_{m=1}^{M_{i}} \beta_{i, m} P\left(\lambda_{i}, m\right)
$$

where $P\left(\lambda_{i}, m\right)$ is defined in (31).

\section{SimUlation ResUlts}

The derived analytic expression for the average BEP has been verified by numerical simulations. All experiments were performed under an environment of perfect equal power control, coherent detection, system bandwidth $W$, perfect channel state information, and a frequency selective Rayleigh fading channel

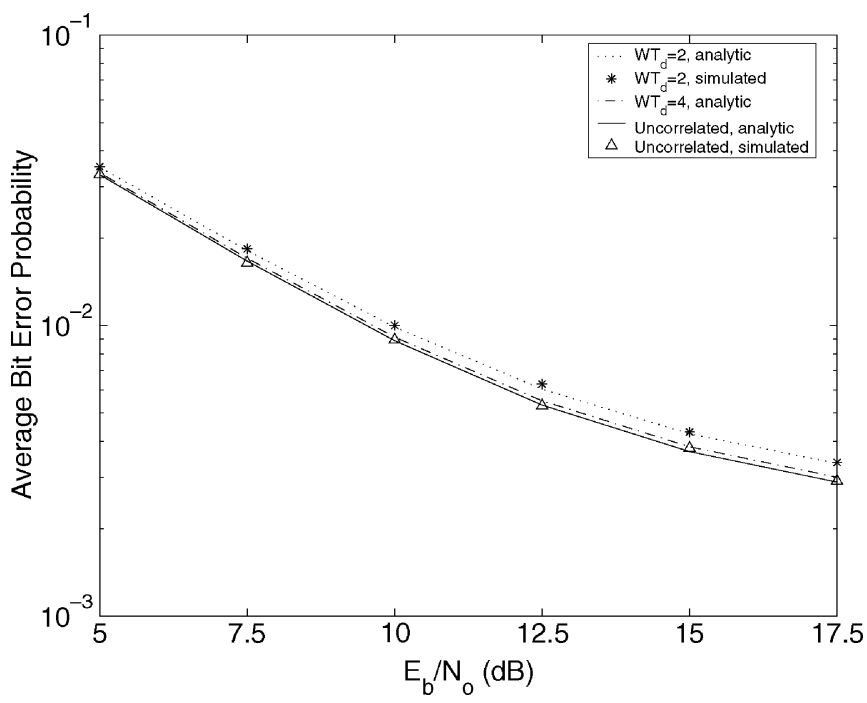

Fig. 2. The average BEP versus $E_{b} / N_{0}$ of an MC-DS-CDMA system with correlated fading when $M=4, N_{c}=32$, and $K=20$, where a set of distinct random sequences is employed to modulate all sub-carriers for each user.

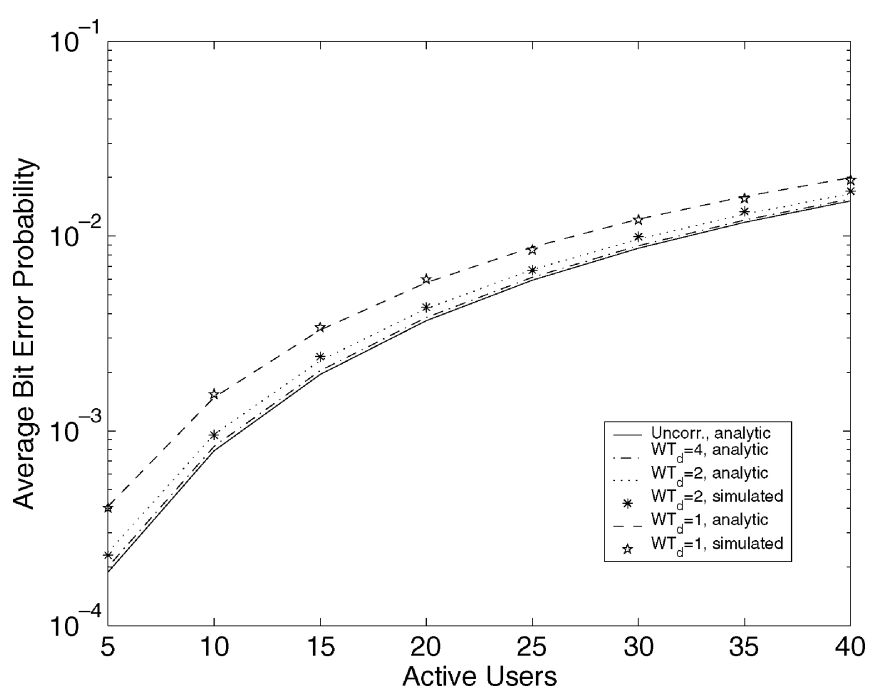

Fig. 3. The average BEP versus the number of active users for an MC-DS-CDMA system with $M=4, N_{c}=32$ and $E_{b} / N_{o}=15 \mathrm{~dB}$, where a set of distinct random sequences is employed to modulate different sub-carriers of a user.

corrupted by AWGN. A WSSUS channel model with exponential MIP and unit energy constraint was adopted. Thus, the channel frequency response correlation between any two subchannels can be determined with $W T_{d}$ in (5) serving as a parameter. To average the effects of random transmission delays and Rayleigh fading, we updated the independently generated $\tau_{k}$ 's and $\mathbf{h}_{k}$ 's every frame with a length of 200 symbols. The Monte Carlo simulation technique was employed so that the simulated BEP had a relative precision within $\sqrt{5} \times 10^{-2}[18]$.

Fig. 2 compares simulated and analytic results of BEP for an MC-DS-CDMA system with $M=4$ sub-carriers by varying the correlation degree among sub-channels with frequency nonselectivity. The system was set to have $K=20$ active users, and a set of $M$ distinct random spreading sequences with a spreading ratio $N_{c}=32$ was assigned to each user for 


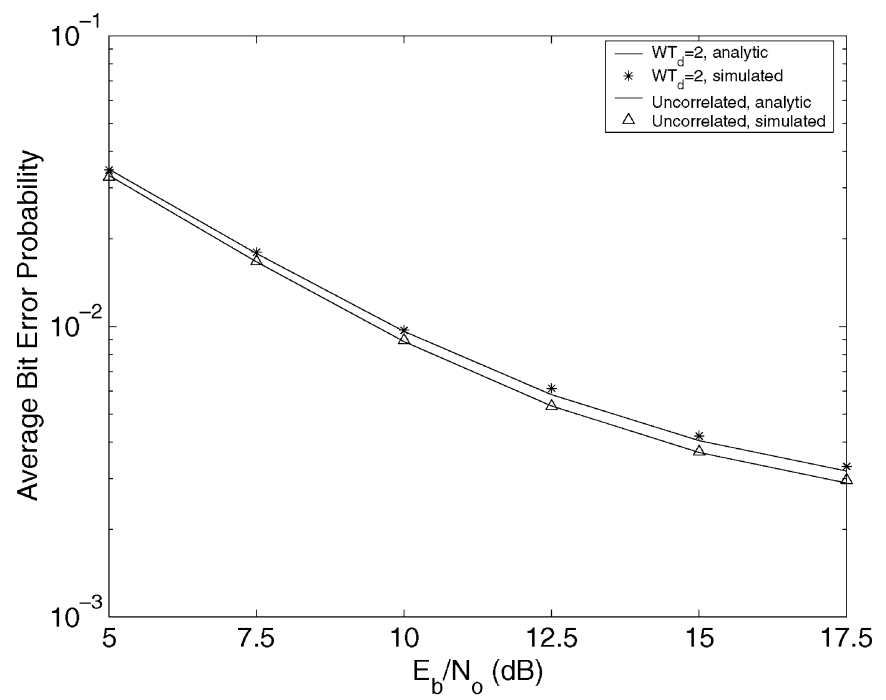

(a)

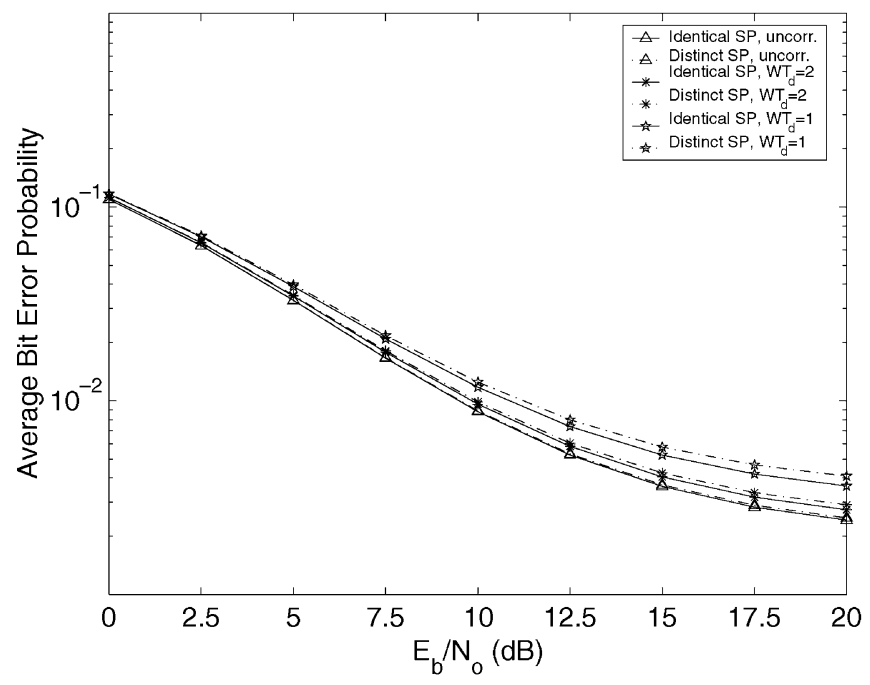

(b)

Fig. 4. (a) The analytic and simulated BEP when a common spreading code is used over all sub-carriers for each user. (b) Comparison of the analytic BEP between two spreading strategies, where the solid line represents the case when a common random spreading code is used over all sub-carriers for each user and the dotted line represents the case when a set of distinct random spreading sequences is employed. The system parameters in (a) and (b) are $M=4, N_{c}=$ 32 , and $K=20$.

modulating each sub-carrier. It is clear that the simulated and analytic results match each other very well. We also see that, as expected, the system with uncorrelated fading has a better performance than that of the correlated case. The degradation becomes apparent when the fading correlation gets severe. Nevertheless, as mentioned in [8], the effectiveness of all diversity branches can still be retained when the correlation is not too significant. As shown in Fig. 2, the performance difference between the curve with uncorrelated fading and that with $W T_{d}=4$ is not very perceptible. A similar conclusion can be drawn from results given in Fig. 3, where the capacity of an MC-DS-CDMA system under several different levels of fading correlation is compared.

Fig. 4 illustrates the effect of different strategies of assigning random spreading sequences on the average BEP of asyn-

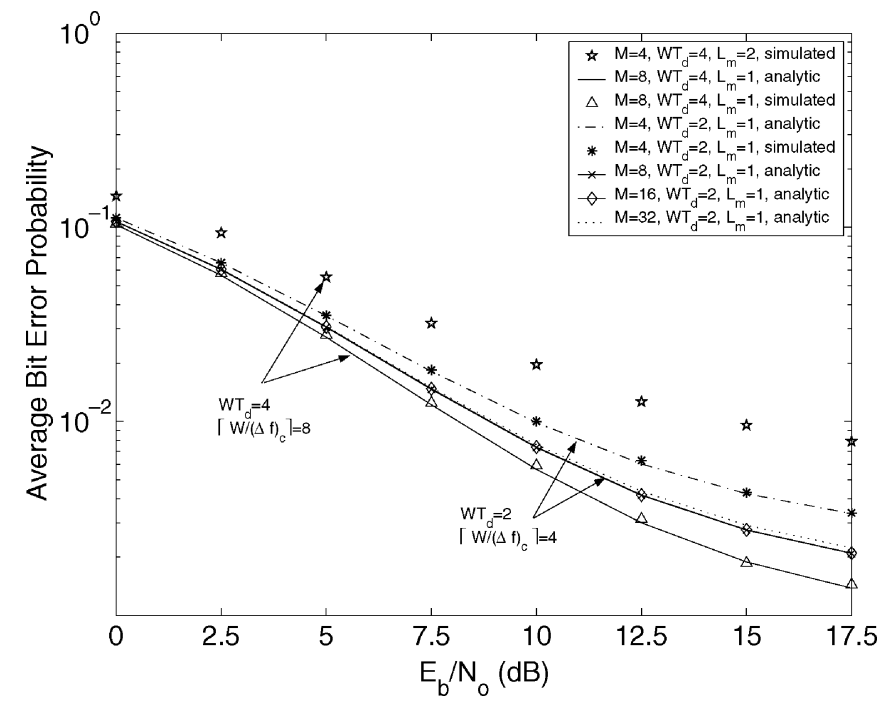

Fig. 5. The BEP of systems with the simultaneous effect of the number of sub-carriers, correlated fading, ICI and multipath interference, where all systems are set to have a common composite ratio $N=128$ and $K=20$.

chronous MC-DS-CDMA systems with $M=4, K=20$, and $N_{c}=32$. Fig. 4(a) shows a good match of our analytic result to the numerical result. In Fig. 4(b), we see that the performance when each user employs a common random spreading sequence over all sub-carriers is better than that when a set of distinct spreading sequences is adopted. This can be explained by the fact that, when a common signature sequence is employed, the statistical correlation among sub-channels has been exploited by the MRC receiver developed in (14) and the impairment caused by ICI is mitigated. In contrast, the information of correlated statistics from other sub-carriers has been zeroed out by the use of distinct uncorrelated random sequences.

Effects of correlated fading, ICI and multipath interference are presented in Fig. 5. The performance of an MC-DS-CDMA system is compared by varying the number of sub-carriers under two channel conditions. First, we consider a channel with $W T_{d}=4$ and $\left\lceil W /(\triangle f)_{c}\right\rceil=8$, i.e., a frequency-selective channel with 8 resolvable propagation paths. For an MC-DS-CDMA system with $M=4$ sub-carriers, there still exists multipath effects with $L_{m}=\left\lceil W / M(\triangle f)_{c}\right\rceil=2$ paths at each sub-channel. Note that there is no exact relationship between channel rms delay spread $T_{d}$ and coherence bandwidth $(\Delta f)_{c}$ [19]. Typically, $T_{d}$ is less than the reciprocal of the channel coherence bandwidth, and it is therefore reasonable to have the parameters assumed in our simulations. In this experiment, correlated fading coefficients for each propagation path over all sub-carriers were generated following the rule described in [9], [12]. By adding the number of sub-carriers to $M=8$ while keeping the same composite spreading ratio (defined by the product of $N_{c}$ and $M$ ) and channel condition, multipath effect is alleviated at the expense of increased fading correlation and ICI. It can be seen from the simulation result that the system with $M=8$ sub-carriers has a better performance, even though the effect of fading correlation and ICI is more severe. This shows that exploiting increased diversity branches compensates the degradation caused by ICI as well as the correlated fading and brings more benefit to the system. 
Finally, we consider a channel with $W T_{d}=2$ and $\left\lceil W /(\triangle f)_{c}\right\rceil=4$. Also shown in Fig. 5, we compare the performance of four systems with $M=4,8,16$ and 32 while keeping an identical composite spreading ratio under this channel condition. In this case, since all systems experience frequency nonselective fading at each sub-channel, not much additional diversity gain can be attained. Simulation results in Fig. 5 show that increasing the number of sub-carriers from $M=4$ to $M=8$ can achieve a potential diversity gain as concluded in [10]. However, because the multicarrier system has exhausted the diversity, increasing the number of sub-carriers from $M=8$ to $M=16$ does not improve the performance much, as we can see that these two curves almost overlap with each other. Notice that, when comparing the curves of $M=16$ and $M=32$, we have a different observation from that given in [10], i.e., we do not obtain an irreducible BEP. The BEP level rises when we keep adding the number of sub-carriers, since the ICI effect begins to emerge.

\section{CONCLUSION}

The effects of long spreading sequences and ICI on the performance of asynchronous multicarrier CDMA systems with correlated fading between sub-channels were investigated in this work. We obtained the MRC filter at the multicarrier system receiver by finding the unconditional covariance matrix of the interference-plus-noise vector. We demonstrated that ICI can be mitigated by assigning a common random spreading sequence over all sub-carriers for a given user with, however, a marginal performance improvement over the scenario when a set of distinct spreading sequences is employed. The analytic expression for BEP was obtained based on Gaussian approximation and was shown to match simulation results very well. Finally, we concluded that it would be desirable to design an MC-DS-CDMA system with a sufficiently large number of sub-carriers to ensure frequency nonselectivity fading. Although this design strategy may lead to more severe ICI and fading correlation, simulations have shown that the degradation is less serious than that of the multipath interference resulting from an insufficient number of sub-carriers.

\section{APPENDIX}

\section{A. Definition of the Block Hadamard Product}

Definition: (Block Hadamard Product) Let $\mathbf{A}$ and $\mathbf{C}$ be two square matrices of dimension $P \times P$ and $Q \times Q$, respectively, with $Q=n \cdot P$ for some positive integer $n$. The matrix $\mathbf{C}$ can be expressed in a block-matrix representation as

$$
\mathbf{C}=\left[\begin{array}{cccc}
(\mathbf{C})_{1,1} & (\mathbf{C})_{1,2} & , \ldots, & (\mathbf{C})_{1, P} \\
(\mathbf{C})_{2,1} & (\mathbf{C})_{2,2} & , \ldots, & (\mathbf{C})_{2, P} \\
\vdots & \vdots & \ddots & \vdots \\
(\mathbf{C})_{P, 1} & (\mathbf{C})_{P, 2} & , \ldots, & (\mathbf{C})_{P, P}
\end{array}\right]
$$

with $(\mathbf{C})_{i, j}$, for $1 \leq i, j \leq P$, denoting the block matrix of size $n \times n$ located at the $(i, j)$ th block location of $\mathbf{C}$. Then, the block Hadamard product of $\mathbf{A}$ and $\mathbf{C}$, denoted by $\mathbf{A} \odot \mathbf{C}$, is defined to be the $Q \times Q$ matrix as follows:

$$
\mathbf{A} \odot \mathbf{C} \triangleq\left[\begin{array}{cccc}
\mathbf{A}_{1,1}(\mathbf{C})_{1,1} & \mathbf{A}_{1,2}(\mathbf{C})_{1,2} & , \ldots, & \mathbf{A}_{1, P}(\mathbf{C})_{1, P} \\
\mathbf{A}_{2,1}(\mathbf{C})_{2,1} & \mathbf{A}_{2,2}(\mathbf{C})_{2,2} & , \ldots, & \mathbf{A}_{2, P}(\mathbf{C})_{2, P} \\
\vdots & \vdots & & \vdots \\
\mathbf{A}_{P, 1}(\mathbf{C})_{P, 1} & \mathbf{A}_{P, 2}(\mathbf{C})_{P, 2} & , \ldots, & \mathbf{A}_{P, P}(\mathbf{C})_{P, P}
\end{array}\right]
$$

In the case of $n=1$, the block Hadamard product degenerates to the standard entry-wise Hadamard product operation.

\section{B. Derivation of (26)}

The covariance matrix of $\mathbf{d}_{k, r}$ given in (25) can be further expressed as

$$
E\left[\mathbf{d}_{k, r} \mathbf{d}_{k, r^{\prime}}^{H}\right]=\frac{1}{N_{c}}\left(\mathbf{1}_{M} \otimes \mathbf{I}_{2}\right)\left(\mathbf{1}_{M} \otimes \mathbf{I}_{2}\right)^{H}
$$

where $\mathbf{1}_{M}$ denotes the $M \times 1$ vector with all entries equal to 1 . Thus, we obtain

$$
\begin{aligned}
E & {\left[\mathbf{d}_{k, m}^{H}\left(\mathbf{R} \odot \mathbf{W}_{m, n}\right) \mathbf{d}_{k, n}\right] } \\
& =\operatorname{tr}\left\{\left(\mathbf{R} \odot \mathbf{W}_{m, n}\right) E\left[\mathbf{d}_{k, n} \mathbf{d}_{k, m}^{H}\right]\right\} \\
& =\frac{1}{N_{c}} \operatorname{tr}\left\{\left(\mathbf{1}_{M} \otimes \mathbf{I}_{2 \times 2}\right)^{H}\left(\mathbf{R} \odot \mathbf{W}_{m, n}\right)\left(\mathbf{1}_{M} \otimes \mathbf{I}_{2 \times 2}\right)\right\} \\
& =\frac{1}{N_{c}} \sum_{p=1}^{M} \sum_{q=1}^{M} \mathbf{R}_{p, q} \cdot \operatorname{tr}\left\{\left(\mathbf{W}_{m, n}\right)_{p, q}\right\} .
\end{aligned}
$$

We see that only the traces of $\left(\mathbf{W}_{m, n}\right)_{p, q}$ are of our particular interest.

\section{Analytic Results of $\left(\mathbf{W}_{m, n}\right)_{p, q}$}

The explicit analytic results of $\left(\mathbf{W}_{m, n}\right)_{p, q}$ defined in (22) can be carried out by considering two scenarios when $m=n$ as well as $m \neq n$, and are provided in the following.

Case I: $m=n$ : This case corresponds to evaluating the diagonal block appearing in (20). Each $2 \times 2$ matrix $\mathbf{B}_{0}-\mathbf{B}_{4}$, representing the corresponding $\left(\mathbf{W}_{m, n}\right)_{p, q}$ shown in Fig. 6(a) is given by

$$
\begin{aligned}
& \mathbf{B}_{0}=\frac{1}{6}\left(\begin{array}{ll}
2 & 1 \\
1 & 2
\end{array}\right) \text { for } p=q=m \\
& \mathbf{B}_{1}=\frac{1}{2 \pi^{2}(m-p)^{2}} \cdot \mathbf{J}_{0} \text { for } p=q \neq m \\
& \mathbf{B}_{2}=\frac{-1}{4 \pi^{2}(m-q)^{2}} \cdot\left(\mathbf{J}_{0}+j \pi(m-q) \mathbf{J}_{1}\right) \text { for } p=m, q \neq m \\
& \mathbf{B}_{3}=\frac{-1}{4 \pi^{2}(m-p)^{2}} \cdot\left(\mathbf{J}_{0}+j \pi(m-p) \mathbf{J}_{1}\right) \text { for } q=m, p \neq m \\
& \mathbf{B}_{4}=\frac{1}{4 \pi^{2}(m-p)(m-q)} \cdot \mathbf{J}_{0} \text { for } p \neq q \neq m .
\end{aligned}
$$




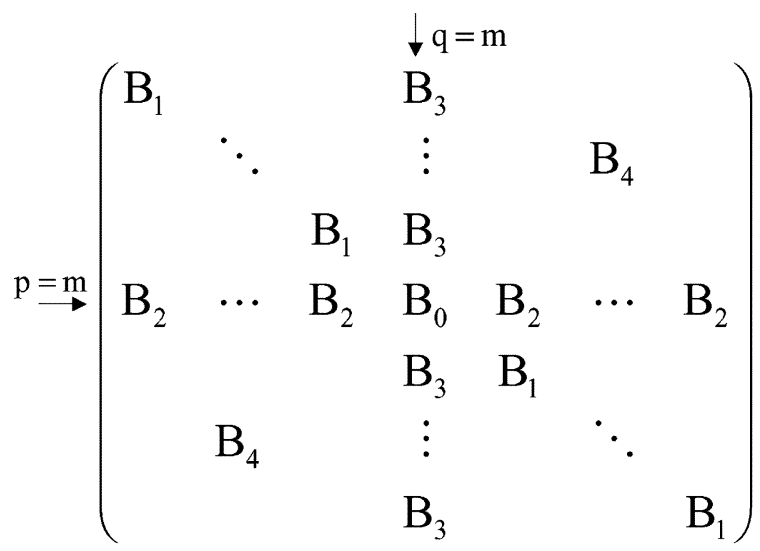

(a) $\mathrm{m}=\mathrm{n}$, Diagonal Block

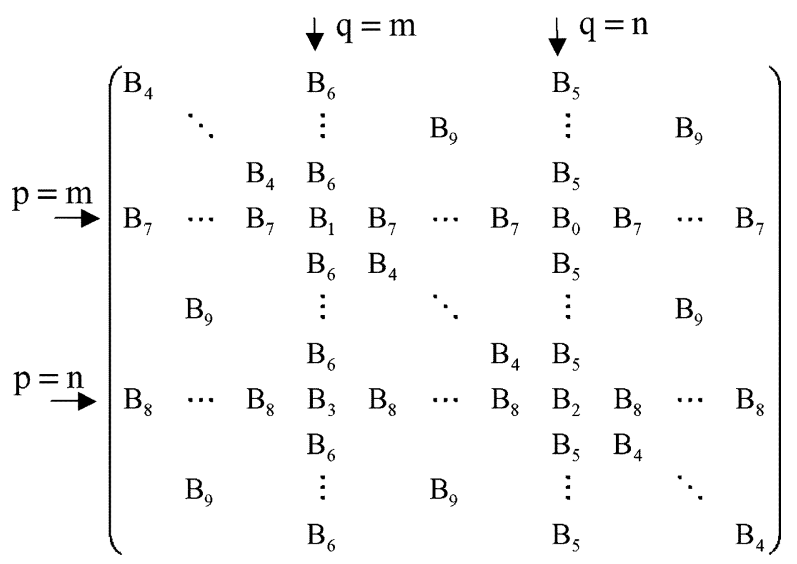

(b) $\mathrm{m} \neq \mathrm{n}$, Off-diagonal Block

Fig. 6. Block matrix $\mathbf{W}_{m, n}$ for (a) $m=n$ and (b) $m \neq n$, where each $\mathbf{B}_{i}$, for $i=0, \ldots,, 9$, represents a possible result of the $2 \times 2$ matrix $\left(\mathbf{W}_{m, n}\right)$ defined in (24).

Case II: $m \neq n$ : Now, consider the off-diagonal block matrix appearing in (20). This requires evaluating the $2 M \times 2 M$ matrix $\mathbf{W}_{m, n}$ for $m \neq n$. Each $2 \times 2$ matrix $\mathbf{B}_{0}-\mathbf{B}_{9}$ representing $\left(\mathbf{W}_{m, n}\right)_{p, q}$ shown in Fig. 6(b) is given by

$$
\begin{aligned}
& \mathbf{B}_{0}=\frac{1}{2 \pi^{2}(m-n)^{2}} \cdot\left(\mathbf{J}_{0}+j \pi(m-n) \mathbf{J}_{2}\right) \text { for } p=m, q=n \\
& \mathbf{B}_{1}=\frac{-1}{4 \pi^{2}(m-n)^{2}} \cdot\left(\mathbf{J}_{0}+j \pi(m-n) \mathbf{J}_{1}\right) \text { for } p=q=m \\
& \mathbf{B}_{2}=\frac{-1}{4 \pi^{2}(m-n)^{2}} \cdot\left(\mathbf{J}_{0}+j \pi(m-n) \mathbf{J}_{1}^{T}\right) \text { for } p=q=n \\
& \mathbf{B}_{3}=\frac{1}{2 \pi^{2}(m-n)^{2}} \cdot \mathbf{J}_{0} \text { for } p=n, q=m \\
& \mathbf{B}_{4}=\frac{1}{4 \pi^{2}(m-p)(n-q)} \cdot \mathbf{J}_{0} \text { for } p=q \neq m \neq n \\
& \mathbf{B}_{5}=\frac{1}{4 \pi^{2}(m-n)(p-n)} \cdot \mathbf{J}_{0} \text { for } p \neq m \neq n, q=n \\
& \mathbf{B}_{6}=\frac{1}{4 \pi^{2}(m-p)(m-n)} \cdot \mathbf{J}_{0} \text { for } p \neq m \neq n, q=m \\
& \mathbf{B}_{7}=\frac{1}{4 \pi^{2}(m-q)(m-n)} \cdot \mathbf{J}_{0} \text { for } p=m, q \neq n \neq m \\
& \mathbf{B}_{8}=\frac{1}{4 \pi^{2}(m-n)(q-n)} \cdot \mathbf{J}_{0} \text { for } p=n, q \neq m \neq n \\
& \mathbf{B}_{9}=\mathbf{0}, \quad \text { otherwise }
\end{aligned}
$$

where the matrices $\mathbf{J}_{0}, \mathbf{J}_{1}$, and $\mathbf{J}_{2}$ appearing in the above are respectively defined as

$$
\mathbf{J}_{0}=\left(\begin{array}{cc}
1 & -1 \\
-1 & 1
\end{array}\right) \quad \mathbf{J}_{1}=\left(\begin{array}{cc}
1 & -1 \\
1 & -1
\end{array}\right) \quad \mathbf{J}_{2}=\left(\begin{array}{cc}
1 & 0 \\
0 & -1
\end{array}\right) .
$$

\section{REFERENCES}

[1] S. Kondo and L. B. Milstein, "Performance of multicarrier CDMA systems," IEEE Trans. Commun., vol. 44, no. 2, pp. 238-246, Feb. 1996.

[2] N. Yee, J. P. Linnartz, and G. Fettweis, "Multi-carrier CDMA in indoor wireless radio networks," in Proc. IEEE PIMRC, Sep. 1993, pp. 109-113.

[3] S. L. Miller and B. J. Rainbolt, "MMSE detection of multicarrier CDMA," IEEE J. Select Areas Commun., vol. 18, no. 11, pp. 2356-2362, Nov. 2000

[4] J. Namgoong, T. F. Wong, and J. S. Lehnert, "Subspace multiuser detection for multicarrier DS-CDMA," IEEE Trans. Commun., vol. 48, no. 11, pp. $1897-1908$, Nov. 2000.

[5] L. Fang and L. B. Milstein, "Successive interference cancellation in multicarrier DS CDMA," IEEE Trans. Commun., vol. 48, pp. 1530-1540, Sep. 2000.

[6] T. M. Lok, T. F. Wong, and J. S. Lehnert, "Blind adaptive signal reception for MC-CDMA systems in Rayleigh fading channels," IEEE Trans. Commun., vol. 47, pp. 464-471, Mar. 1999.

[7] H. Liu and H. Yin, "Receiver design in multicarrier direct-sequence CDMA communications," IEEE Trans. Commun., vol. 49, no. 8, pp. 1479-1487, Aug. 2001.

[8] M. Schwartz, W. R. Bennett, and S. Stein, Communication Systems and Techniques. New York: IEEE Press, 1996.

[9] W. C. Y. Lee, Mobile Communications Engineering. New York: McGraw-Hill, 1982

[10] R. E. Ziemer and N. Nadgauda, "Effect of correlation between subcarriers of an MCM/DSSS communication system," in Proc. IEEE VTC'96, vol. 1, 1996, pp. 146-150.

[11] W. Xu and L. B. Milstein, "Performance of multicarrier DS CDMA systems in the presence of correlated fading," in Proc. IEEE VTC'97, vol 3, May 1997, pp. 2050-2054.

[12] - "On the performance of multicarrier RAKE systems," IEEE Trans Commun., vol. 49, no. 10, pp. 1812-1823, Oct. 2001.

[13] S. Hara and R. Prasad, "Design and performance of multicarrier CDMA system in frequency-selective Rayleigh fading channels," IEEE Trans. Veh. Technol., vol. 48, no. 5, pp. 1584-1595, Sep. 1999.

[14] X. Gui and T. S. Ng, "Performance of asynchronous orthogonal multicarrier CDMA system in frequency selective fading channel," IEEE Trans. Commun., vol. 47, no. 7, pp. 1084-1091, Jul. 1999.

[15] D. Guo, S. Verdú, and L. K. Rasmussen, "Asymptotic normality of linear multiuser receiver outputs," IEEE Trans. Inform. Theory, vol. 48, no. 12 pp. 3080-3095, Dec. 2002.

[16] S.-M. Tseng and M. R. Bell, "Asynchronous multicarrier DS-CDMA using mutually orthogonal complementary sets of sequences," IEEE Trans. Commun., vol. 48, no. 1, pp. 53-59, Jan. 2000.

[17] S. Verdú, Multiuser Detection. Cambridge, U.K.: Cambridge Univ. Press, 1998

[18] M. C. Jeruchim, P. Balaban, and K. S. Shanmugan, Simulation of Communication Systems: Modeling, Methodology, and Techniques, 2nd ed. New York: Kluwer Academic/Plenum, 2000.

[19] T. S. Rappaport, Wireless Communications: Principles and Practice. Englewood Cliffs, NJ: Prentice-Hall, 1996.

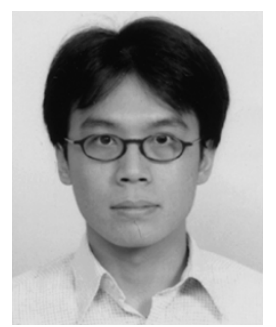

Feng-Tsun Chien (S'02) received the B.S. degree from the National Tsing Hua University, Hsinchu, Taiwan, in 1995, the M.S. degree from the National Taiwan University, Taipei, in 1997, and the Ph.D. degree from the University of Southern California, Los Angeles, in 2004, respectively, all in electrical engineering.

His research interests include signal processing aspects of communications, cross-layer designs, multicarrier CDMA and MIMO-OFDM systems. 


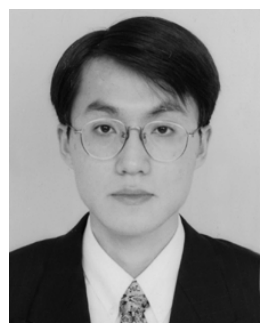

Chien-Hwa Hwang (S'01-M'04) received the B.S. and M.S. degrees from the National Taiwan University, Taipei, in 1993 and 1995, respectively, and the Ph.D. degree from the University of Southern California, Los Angeles, in 2003, all in electrical engineering.

In August 2003, he joined the Institute of Communications Engineering of the National Tsing Hua University, Taiwan, as an Assistant Professor. His research interests include multiuser detection, multicarrier communications and graph theory.

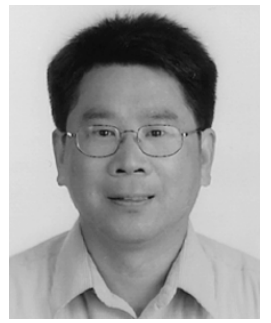

C.-C. Jay Kuo (S'83-M'86-SM'92-F'99) received the B.S. degree from the National Taiwan University, Taipei, in 1980 and the M.S. and Ph.D. degrees from the Massachusetts Institute of Technology, Cambridge, in 1985 and 1987, respectively, all in electrical engineering.

He was Computational and Applied Mathematics (CAM) Research Assistant Professor in the Department of Mathematics at the University of California, Los Angeles, from October 1987 to December 1988. Since January 1989, he has been with the Department of Electrical Engineering-Systems and the Signal and Image Processing Institute at the University of Southern California, Los Angeles, where he currently has a joint appointment as Professor of Electrical Engineering and Mathematics. His research interests are in the areas of digital signal and image processing, audio and video coding, multimedia communication technologies and delivery protocols, and embedded system design. He has guided about 60 students to their $\mathrm{Ph} . \mathrm{D}$. degrees and supervised 15 postdoctoral research fellows. He is co-author of seven books and more than 700 technical publications in international conferences and journals.

Dr. Kuo is a Fellow of IEEE and SPIE and a member of ACM. He is Editor-in-Chief for the Journal of Visual Communication and Image Representation, and Editor for the Journal of Information Science and Engineering and the EURASIP Journal of Applied Signal Processing. He was on the Editorial Board of the IEEE Signal Processing Magazine in 2003-2004. He served as Associate Editor for IEEE TRANSACTIONS ON IMAGE PROCESSING in 1995-1998, IEEE TRANSACTIONS ON CIRCUITS AND SYSTEMS FOR VIDEO TECHNOLOGY in 1995-1997 and IEEE TRANSACTIONS ON SPEECH AND Audio PROCESSING in 2001-2003. He received the National Science Foundation Young Investigator Award (NYI) and Presidential Faculty Fellow (PFF) Award in 1992 and 1993, respectively. 